\title{
Die Psychiatriereform war nur eine Modernisierung
}

\author{
The Reformation of Psychiatry was Only a Modernization
}

Kontra: Manfred Bauer

Bibliografie

DOI $10.1055 / \mathrm{s}-2007-970817$

Psychiat Prax 2007; 34:

215-217 @ Georg Thieme

Verlag KG Stuttgart · New York .

ISSN 0303-4259

Korrespondenzadressen

Dr. med. Sebastian StierI

Ltd. Arzt am NLKH Lüneburg

Am Wienebütteler Weg 1

21339 Lüneburg

Sebastian.Stierl@nlkh-

lueneburg.Niedersachsen.de

Prof. Dr. Manfred Bauer

Keltenring 85

63128 Dietzenbach

Manfredbauerof@aol.com

\section{Pro \\ Proch}

„Ich glaube, dass es ganz wichtige Gesichtspunkte sind, die von jedem Sozialpsychiater zu bedenken sind, dass er weiß, unter welchen gesellschaftlichen Bedingungen er arbeitet und die Tatsachen zu erkennen lernt, die Sozialpsychiatrie in den letzten Jahren möglich gemacht hat..., um einkalkulieren zu können, dass bei einer Veränderung der ökonomischen Lage diese sozialpsychiatrischen Ansätze als ein modischer Firlefanz abgetan werden können und es einen Rückzug der Psychiatrie auf alte Positionen und alte Organisationsmodelle geben kann“ (1971).

Der Reformbegriff wird zunehmend zur Verschleierung des Sozialabbaus missbraucht. Es scheint deshalb sinnvoll, die Psychiatriereform auf ihre Substanz zu überprüfen.

Dabei definiere ich Reform als politischen Transformationsprozess der Anpassung gesellschaftlicher Verhältnisse (hier der psychiatrischen Versorgung) im Interesse einer demokratischen Mehrheit zur nachhaltigen Verbesserung der Lebensbedingungen unter besonderer Berücksichtigung von Minderheiten und Schwachen (hier der Schwer- und chronisch psychisch Kranken). Davon unterscheide ich die Modernisierung. Diese findet ebenfalls als Anpassung an sich verändernde Verhältnisse statt, hier aber vorrangig „nach dem neuesten Stand der Wissenschaft". Begriffe wie „Markt“, „Effektivität“, „Konkurrenz“ und "Individualisierung der Lebensgestaltung“ lassen dabei die grundsätzlich ideologische Ausrichtung an den Maßstäben einer neoliberal-kapitalistischen Gesellschaft erkennen. Während „Staat" und „Solidarität“ als verstaubt und überholt gelten, wird „privat“ und „Profit“ zwar nicht unbedingt als gerecht, sicher aber als modern assoziiert.

Ausgehend von der Bestandsaufnahme „teilweise elender, menschenunwürdiger Verhältnisse“ (Zwischenbericht der Enquete 1973) sollte die Psychiatrie am Ende der notwendigen Reform bedarfsgerecht, gemeindeorientiert und seelisch Kranke endlich mit körperlich Kranken gleichgestellt sein. Die Expertenkommission hatte 1988 zu ihrer Umsetzung konkretere Schritte empfohlen.

Die kritische Bilanz ist ernüchternd. Unzulängliche gesetzliche Präzisierungen und fehlende Durchsetzungsinstrumente führen aufgrund mangelnder Verbindlichkeit zu unterschiedlichen Standards und Versorgungsniveaus in den Regionen (z. B. Zwangseinweisungsraten, Rehabilitationsmöglichkeiten, Hilfebedarfskriterien). Das Regionalisierungskonzept als Prinzip kollektiver Verantwortung bleibt vom guten Willen der Beteiligten abhängig, schlimmstenfalls bildet es den Rahmen für einen Kampf um „Kunden“, um das Überleben der Einrichtung „am Markt“. Fachliche Standards werden im Gesetzgebungsverfahren soweit verzerrt, bis sie schließlich als „Leistungsverhinderungsgesetze“ (z. B. Soziotherapie, Ambulante Psychiatrische Pflege) in Kraft treten.

Der Bettenabbau in den psychiatrischen Anstalten verschleiert die Tatsache, dass in der Regel neben der Schaffung neuer Betten in psychiatrischen Abteilungen an Allgemeinkrankenhäusern der Ausbau privater Heimplätze insgesamt zu einem Anstieg psychiatrischer Betten in der Region und somit zu einer Verstärkung der stationären Versorgung führt (von wegen: ,ambulant vor stationär“!). Mit der Privatisierung von Landeskrankenhäusern ist die Hoffnung auf weitere Bettenreduzierung endgültig tot: wer wird schon die Kuh schlachten, die man gekauft hat, um sie zu melken?

Bei dieser Rechnung bleibt die ungeheuerliche Verdopplung forensischer Betten innerhalb der letzten 10 Jahre noch unberücksichtigt.

„Personenzentrierung“, ursprünglich entstanden in Abgrenzung zur "Institutionszentrierung“, pervertiert in der Praxis viel zu oft (z.B. in Lüneburg) zur Legitimierung entwürdigender Hilfebe- 
darfskontrollen und ihrer restriktiven Umsetzung in Fachleistungsstunden. Unmerklich verschieben sich unter Rationierung und Kostendruck die Patienten der Psychiatrie in andere Zuständigkeiten: hier in die Forensik, dort in die Obdachlosigkeit. Für die weniger Störenden locken die gepflegten Betten von Psychosomatik und Psychotherapie. Die postmoderne Individualisierung und Entstaatlichung spiegelt sich in der Psychiatrie in Form von Entsolidarisierung, Deregulierung und Deinstitutionalisierung. Psychiatrie entwickelt sich unter diesen Bedingungen vom „öffentlichen Gut“ zur „warenförmigen Dienstleistung“ (2005).

Auch in der Klinik halten sich die Fortschritte, z. B. bei der Behandlung akuter Psychosen, in Grenzen. An den Milieus der Aufnahmestationen sind die Erkenntnisse der biopsychosozialen Forschung vorbeigegangen: zur Reizabschirmung und Entängstigung werden nach wie vor gleich (un-)wirksame Medikamente missbraucht, um konzeptionelle Ideenlosigkeit, Personalabbau und Qualifikationsdefizite zu kompensieren. Die Nebenwirkungen sind zwar nicht mehr so auffällig, dafür aber nicht weniger schwerwiegend. Bei qualitätsgesichertem Personalabbau zerrinnt uns die PsychPV zwischen den Fingern. Nach außen reden wir von Psychotherapie und Beziehung und in Wirklichkeit reduziert sich der direkte Patientenkontakt bei den Ärzten mittlerweile auf weniger als die Hälfte ihrer Arbeitszeit. Immerhin herrschen in der Psychiatrie keine elenden, menschenunwürdigen Verhältnisse mehr. Auch forensische Stationen sind heute hell und geschmackvoll eingerichtet, die Hightech-Überwachung dezent verkleidet - und auch der personellen Kontinuität in der Therapie wird hier Rechnung getragen...

Nach der Entlassung stauen sich die Patienten vor den psychotherapeutischen Praxen, während psychiatrische Kassenarztsitze zunehmend verwaisen. Mit den weiter boomenden (Alten-)Heimen, den privatisierten Krankenhäusern und dem Wegbrechen der psychiatrischen „Tante-Emma-Praxen“ ist die Psychiatrie in der neoliberalen Wirklichkeit angekommen. Psychiatrisches Handeln wird in nie da gewesener Weise einer allumfassenden Ökonomisierung unterworfen, Beziehungen in Minutenwerte und Teilleistungseinheiten zerhackt. Die Abkehr von der staatlich garantierten Funktion sozialer Sicherung in Richtung auf eine gewinnorientierte, betriebswirtschaftliche Ausrichtung des Sozialen trifft auch in der Psychiatrie - wen wundert's - vorrangig chronisch Kranke.

Da die Welt uns nicht den Gefallen getan hat, sich trotz unseres entschieden moralischen Anspruchs in eine gerechtere zu verwandeln, werden wir die bestehende Ungerechtigkeit wohl als gegeben anerkennen. Psychiatrie wäre künftig als Teil des medizinischen Dienstleistungsbetriebes zu begreifen, wo sie sich als Ware konkurrierend auf dem expandierenden Gesundheitsmarkt behaupten muss.

Die gesellschaftlichen Bedingungen haben sich seit 1971 gewaltig verändert. Ein Zurück, wie es M. Richartz befürchtete, wird es nicht geben. Allerdings ist etwas anderes herausgekommen, als von der DGSP und den Reformern ursprünglich geplant war. Bei unbestritten positiven Veränderungen in Teilbereichen (Abteilungen, Tageskliniken, Betreutes Wohnen etc.) sehen wir keine Reform, sondern eine konsequente Modernisierung der Psychiatrie.

\section{Kontra}

Auf das in einer Rundfunkdiskussion zum Thema Sozialpsychiatrie zwischen H. E. Richter, E. Wulff, K. Dörner, N. Pörksen und Th. Winkler gesprochene Wort von Mark Richartz, zu jener Zeit Assistenzarzt an der Medizinischen Hochschule Hannover, jetzt Emeritus in Maastricht, will ich zur Verdeutlichung des damaligen Sprachduktus junger und sich als „links“ verstehender angehender Psychiater ebenfalls mit einer Sentenz beginnen, die im Sonderband 60 der Zeitschrift Das Argument- Kritik der bürgerlichen Medizin - unter dem Titel „Angepasste Psychiatrie als Psychiatrie der Anpassung“ (1970) gedruckt wurde: „Solange das Verhältnis der Gesellschaft zu ihren Verrückten unreflektiert und seine Kritik ohne praktische Konsequenz für das Verhalten der Therapeuten bleibt, werden psychiatrische Kliniken Agenturen zur Stabilisierung und Konkretisierung eben dieser Verhältnisse bleiben“.

So haben Mark Richartz und Manfred Bauer (1972) damals theoretisiert, weil es im Elfenbeinturm einer Universitätsklinik zu wenig Praktisches zu tun gab. Erst ein mehrmonatiger Aufenthalt am Maudsley Hospital bei Douglas Bennett und John Wing gab entscheidende neue Impulse in Sachen Psychiatrie. Nicht zuletzt dazu, sich so auszudrücken, dass auch andere es verstehen konnten. Seitdem bin ich davon überzeugt, dass der Psychiatrie als medizinischem Fach mit dem gesellschaftlichen Auftrag, psychische Krankheiten zu erkennen, wenn möglich, sie zu heilen, zu bessern oder wenigstens das damit verbundene Leid zu mildern, nicht mit großartigen, gesellschaftskritischen Konzepten gedient ist, sondern mit kleinen Hilfen für die Patienten und ihre Angehörigen und kleinen professionellen Schritten in die richtige Richtung, wobei man über die Richtung durchaus streiten kann und sollte.

Soll man die Psychiatrie z.B. als konstitutiven Teil der allgemeinen Medizin begreifen und sie dann konsequenterweise an Allgemeinkrankenhäusern als ein Fach unter anderen betreiben? Das hätte 1973 geheißen sofort mit dem Abriss der Anstalten und dem Neubau derartiger Abteilungen an Allgemeinkrankenhäusern zu beginnen; oder soll man erst mit viel Geld die im Zwischenbericht (1973) der Psychiatrie-Enquete-Kommission angeprangerten „menschenunwürdigen Verhältnisse“ in den psychiatrischen Sonderkrankenhäusern beseitigen, sie also modernisieren mit der Konsequenz, sie damit auch für die nächste und übernächste Generation zu erhalten?

Bekanntlich ist diese Richtungsfrage bis heute nicht beantwortet, mit der Folge, dass bislang nur eine einzige Psychiatrische Anstalt definitiv geschlossen wurde (PKH Merzig/Saarland), während sich alle anderen Fachkrankenhäuser verkleinert und durchsaniert haben. In diesem Punkt hat Stierl recht: was die psychiatrischen Fachkrankenhäuser angeht, ist die Psychiatriereform auf halbem Wege stecken geblieben, wie vor einiger Zeit das Deutsche Ärzteblatt titelte und sich dabei auch auf einige unserer Nachbarländer bezog.

Polemisch zugespitzt könnte man sagen, die „Modernisierer“ blieben in den Anstalten unter sich, die „Reformer“ gingen in die Gemeinde. 170 Abteilungen an Allgemeinkrankenhäusern legen davon inzwischen Zeugnis ab, während es im Jahr 1971 gerade eben mal 21 waren, alle ohne Versorgungsverpflichtung und die meisten neuropsychiatrisch ausgerichtet, wie es damals Usus war. Heutzutage tragen sie zu mehr als $50 \%$ zur stationären Versorgung bei und leisten allein durch ihre Existenz mehr Antistigmaarbeit als alle in den letzten 25 Jahren zu diesem Thema durchgeführten wissenschaftlichen Tagungen. Von der eigentlichen gemeindepsychiatrischen Arbeit im Verbund und vor Ort ganz abgesehen. 
Dass die Psychiatrie - wenn auch nicht überall - inzwischen in der Gemeinde angekommen ist, nenne ich Reform. Sie ist in den Regionen am besten gelungen und am weitesten fortgeschritten, in denen die psychiatrischen Betten in einem Allgemeinkrankenhaus mit regionaler Pflichtversorgung stehen und die außerstationären Einrichtungen und Dienste so eng wie möglich miteinander und mit der Psychiatrischen Abteilung selbst verflochten sind, am besten nicht nur institutionell, sondern auch personell. Da und dort ist dieses Kunststück gelungen eher in kleinen und mittleren Städten. Auf dem flachen Land und in großstädtischem Milieu ist die Lage naturgemäß schwieriger.

Es haben sich aber nicht nur Strukturen der psychiatrischen Versorgung verändert, sondern, was viel wichtiger ist, im Laufe der letzten 25 oder 30 Jahren ist es auch - langsam, aber stetig - zu einem tief greifenden Mentalitätswandel des gesamten therapeutischen Personals gekommen. Man könnte dem die Überschrift geben: vom patriarchalischem Überwachungs- und Disziplinierungssystem zu einer therapeutischen Partnerschaft. Hierzu hat vieles und haben viele beigetragen, letztlich auch die pharmazeutische Industrie durch die Entdeckung und Weiterentwicklung der Psychopharmaka. Hinzu kamen neue und im psychiatrischen Alltag praktikable Psychotherapieverfahren, die es erlaubten kustodiale Positionen zu verlassen und therapeutische einzunehmen.

Wer wie meine Generation der heute knapp 70-Jährigen in den 60er-Jahren anfing, sich für die Psychiatrie als Fach zu interessieren, fand eine trostlosen Landschaft vor, in der nicht nur „teilweise“, sondern durchgängig „elende und menschenunwürdige Verhältnisse“ herrschten. Gewalt unter den Patienten war an der Tages- und Tischordnung, insbesondere dann, wenn das Mittagessen in hässlichen Kübeln auf die Station gebracht wurde und sich die körperlich Stärksten gegen die Schwächeren mit Brachialgewalt durchsetzten. Gelegentlich trauten sich die kräftigsten Pfleger nur gemeinsam und mit einer vor sich hergetragenen Matratze in den Speiseraum, um dort den Streit zu schlichten. Aber auch disziplinarische und willkürliche Gewalt des Pflegepersonals gegen die Patienten gehörte zum Stationsalltag, insbesondere dann, wenn die Ärzte sich nach der Visite in ihre außerhalb der Station gelegene Zimmer zurückzogen, um erst am folgenden Tag wieder zu erscheinen. Man lese nur das Buch von Frank Fischer (1969) oder die Studie von Hemprich und Kisker „Die Herren der Klinik“ (1968), die als verdeckte teilnehmende Beobachtung in der Heidelberger Universitätsklinik durchgeführt wurde, und vergleiche die damaligen mit den heutigen Verhältnissen. Nein, das ist keine „Modernisierung nach dem neuesten Stand der Wissenschaft“, wie uns Stierl weismachen will, sondern ein grundlegender Gesinnungswandel und eine Haltungsänderung bei dem heute amtierenden Personal. Den Kolleginnen und Kollegen mit der „Gnade der späten Geburt" ist nicht vorzuwerfen, dass sie sich leicht vertun bei der Bewertung der jetzigen Verhältnisse, haben sie doch glücklicherweise bei ihrem Eintritt in den Beruf nichts anderes kennen gelernt als das, was die vorige Generation ihnen hinterlassen hat. Da ist keineswegs alles gut, und Stierl hat zu Recht den Finger in manche Wunden gelegt. Aber Vieles ist so schlecht nicht, wie er es darstellt. Gleichwohl lohnt es sich immer, dafür zu kämpfen, dass die (psychiatrische) Welt noch besser wird. Erfahrungsgemäß fängt man damit am besten hinter und vor der eigenen Haustür gleichzeitig an.

PS:

Aber natürlich hat Mark Richartz recht.

\section{Literatur}

Richartz $M$ et al. Was ist Sozialpsychiatrie? (Interview) Soz Psych Inf 1971; 5: 57-93

Fülberth G. G Strich - Kleine Geschichte des Kapitalismus. Köln: Papyrossa, 2005: 15

Bauer M, Richartz M. Angepasste Psychiatrie als Psychiatrie der Anpassung. Das Argument 60 - Kritik der bürgerlichen Medizin. Berlin: 1970: $152-162$

Richartz M, Bauer M. Zur Ideologie der „Arbeit“ in der Sozialpsychiatrie. In: Dörner K, Plog U (Hrsg): Sozialpsychiatrie - Psychisches Leiden zwischen Integration und Emanzipation. München: Luchterhand, 1972: $61-69$

Bauer M. Streifzüge durch die englische Psychiatrie. Soz Psych Inf 1973; Heft 14/15: 1- 164

Hemprich RD, Kisker KP. Die Herren der Klinik und ihre Patienten. Nervenarzt 1968; 39: 433

Fischer F. Irrenhäuser- Kranke klagen an. München: Desch, 1969

Bauer M. Reform als soziale Bewegung: Der „Mannheimer Kreis“ und die „Gründung der Deutschen Gesellschaft für soziale Psychiatrie“. In: Kersting FW (Hrsg): Psychiatrie - Reform als Gesellschaftsreform. Paderborn: Schöningh Verlag, 2003

Häfner H. Die Psychiatrie-Enquete - historische Aspekte und Perspektiven. In: Aktion Psychisch Kranke (Hrsg): 25 Jahre Psychiatrie-Enquete. 2001: $72-102$

Bauer M. Woher wir kommen, wo wir stehen, wohin wir gehen (sollten). Zur Entwicklung der Sozialpsychiatrie in der Bundesrepublik Deutschland. In: Hoffmann-Richter U, Haselbeck H, Engfer R (Hrsg): Sozialpsychiatrie vor der Enquete. Bonn: Psychiatrie Verlag, 1997: 109-121

Bauer M. Von der Schwierigkeit der Gemeindepsychiatrie mit sog. forensischen Patienten. Psych Pflege 1996; 2: $107-111$

Stierl S. Das Ende der Psychiatrie-Reform. Soziale Psychiatrie 2005; $1: 4$ 8 